\title{
Im Zeitalter moderner Stents: Sind die neueren P2Y12-Inhibitoren wirklich besser?
}

\section{Die Leitlinien favorisieren nach einer perkutanen Koronarintervention den Plättchenhemmer Ticagrelor gegenüber Clopidogrel. Eine auf dem ESC- Kongress präsentierte Registerstudie stellt diese Empfehlung nun infrage.}

n den aktuellen Leitlinien der Europäischen Kardiologie-Gesellschaft wird dem neueren P2Y12-Inhibitor Ticagrelor bei Patienten mit akutem Koronarsyndrom (ACS) als Partner zu ASS klar der Vorzug gegenüber Clopidogrel gegeben. Auch in dem gerade erst auf dem ESC-Kongress vorgestelltem „Update zur dualen Antiplättchentherapie" ist diese Präferenz als Klasse-I-Empfehlung fest verankert.

Nun kommt diese Empfehlung durch eine auf demselben Kongress präsentierten Registerstudie namens CHANCE DAPT direkt ins Wanken. In dieser Real-World-Analyse stellte sich nämlich heraus, dass das Risiko-Nutzen-Verhältnis für Ticagrelor schlechter ausfällt als für Clopidogrel. Müssen die Leitlinien überdacht werden?

\section{Leitlinien favorisieren} eindeutig Ticagrelor

Begründung für die Bevorzugung von Ticagrelor sind die Ergebnisse der randomisierten PLATO-Studie. Der neue P2Y12-Inhibitor stellte sich hier als deutlich potenter in der Vermeidung ischämischer Ereignisse heraus als Clopidogrel. Allerdings wurden damals hauptsächlich Metallstents oder Drug Eluting Stents (DES) der älteren Generation verwendet. Die heutigen DES gehen bekanntermaßen mit einem deutlich geringerem Risiko für Stentthrombosen einher.

Diese Tatsache hatte Dr. Clemens von Birgelen und Kollegen zum Nachdenken gebracht. Ist Ticagrelor in Zeiten der modernen DES überhaupt noch überlegen? Seine Arbeitsgruppe hat die PCI-Ergebnisse in der Zeit vor dem Wechsel auf ein Ticagrelor-basiertes DAPT-Regime mit der Zeit danach verglichen (Dezember 2012 bis April 2014 vs. Mai 2014 bis August 2015). Daten von über 2.000 Patienten wurden ausgewertet.

\section{Real-World-Daten lassen zweifeln}

Demnach scheint Ticagrelor ischämische Ereignisse nach einer Stentimplantation nicht wirksamer verhindern zu können als Clopidogrel. Die Herzinfarktund Schlaganfall-Raten betrugen jeweils 2,8 vs. $2,4 \%$ und 1,1 vs. $0,3 \%$. Die Gesamtsterblichkeit war ebenfalls vergleichbar (2,9 vs. $2,0 \%)$.

Auf der anderen Seite war das Risiko für Blutungskomplikationen unter Ticagrelor deutlich erhöht (2,7 vs. 1,2\%). In einer Propensity-Score-MatchingAnalyse fiel dieses fast dreifach höher aus (Hazard Ratio: 2,75).

\section{Nettobenefit für Clopidogrel besser}

Die Nettobilanz - also die gemeinsame Betrachtung von Todesfällen, Herzinfarkten, Schlaganfällen und schweren Blutungen - fiel somit zum Nachteil von Ticagrelor aus (7,8 vs. $5,1 \%)$.

Und das obwohl in der Ticagrelor-Periode häufiger der radiale Zugang, weniger Glyoprotein IIb/IIIa-Inhibitoren und mehr Protonenpumpenhemmer zum Einsatz gekommen seien, betonte von Birgelen. „Ich hoffe, die Gesellschaften prüfen diese Daten sorgfältig", äußerte sich der in Enschede tätige Kardiologe auf einer ESC-Hotline-Session. Für ihn sind die CHANCE-DAPT-Daten „Teil eines Mosaiks“ an kürzlich veröffentlichten Studien, in denen sich ebenfalls kein Vorteil für Ticagrelor ergeben habe, wie die randomisierte TOPIC-Studie und eine vorläufige Auswertung des SCAAR2-Registers.

\section{Aber: Registerstudie mit Limitationen}

Trotz allem ist bei der Interpretation der CHANCE-DAPT-Studie Vorsicht geboten. Es handelt sich um keine randomisierte Studie, sodass immer die Gefahr eines Selektionsbias besteht. So weist auch von Birgelen darauf hin, dass die
Patienten in der Ticagrelor-Periode im Schnitt älter waren. Das Blutungsrisiko sei aber auch in der Propensity-Scoreadjustierten Analyse mit Ticagrelor deutlich höher ausgefallen.

In CHANCE DAPT sind zudem jegliche Patienten mit ACS in die Auswertung eingegangen, wohingegen in der PLATO-Studie nur Patienten mit einem mittleren bis hohem Risiko behandelt wurden.

\section{Forderung nach randomisierter Studie}

Relativiert werden die CHANCE-DAPTDaten auch durch die Ergebnisse des SWEDEHEART-Registers. In dieser Analyse hat sich die Überlegenheit von Ticagrelor in der PLATO-Studie bestätigt.

Nach Ansicht von Birgelen sollte Ticagrelor deshalb momentan nach wie vor der Vorzug gegeben werden. Aber es sei an der Zeit, eine randomisierte Studie aufzulegen, die die Wirksamkeit und Sicherheit beider Plättchenhemmer unter den heutigen Rahmenbedingungen vergleicht. Allerdings sei Clopidogrel mittlerweile günstig und es deshalb nicht im Interesse der Industrie, eine solche Studie zu initiieren. Der Kardiologe hofft deshalb, dass öffentliche Gesundheitsorganisationen und Krankenkassen sich darum bemühen werden.

Veronika Schlimpert

Quelle: Hot Line: Late-Breaking Registry Results 1 "Clopidogrel or Ticagrelor in Acute Coronary Syndrome Patients Treated With Newer-Generation Drug-Eluting Stents: CHANGE DAPT", ESCKongress, am 29.8.2017 in Barcelona

\section{\) ONLINE-TIPP}

\section{Kardiologie.org}

Erstmals wurde der Xience-Stent - der Big Player unter den medikamentenbeschichteten Stents - in einem Direktvergleich mit einem Konkurrenten geschlagen. Die Studie wurde beim ESC-Kongress in Barcelona präsentiert.

www.kardiologie.org/ultraduennerstent-macht-klassenprimus-konkurrenz-/15000012 